\title{
First Results from the Online Radiation Dose Monitoring System in ATLAS Experiment
}

Igor Mandića ${ }^{a}$ V. Cindro ${ }^{a}$, M. Deliyergiyev ${ }^{a}$, A. Gorišek ${ }^{a}$, G. Kramberger ${ }^{a}$, M. Mikuž ${ }^{a}, b$, J. Hartert ${ }^{c}$, S. Franz ${ }^{d}$, I. Dawson ${ }^{e}$, P. Miyagawa ${ }^{e}$, L. Nicolas ${ }^{e}$ on behalf of ATLAS collaboration ${ }^{a J} J$ žef Stefan Institute, Ljubljana, Slovenia, ${ }^{b}$ Faculty of Mathematics and Physics, University of Ljubljana, Slovenia, cPhysikalisches Institut Universität Freiburg, Germany, ${ }^{2} C E R N$, Geneva, Switzerland, ${ }^{e}$ University of Sheffield, UK igor.mandic@ijs.si

- detectors and electronics in ATLAS experiment will be exposed to large doses of radiation: TID $>100 \mathrm{kGy}, \Phi_{\text {eq }}>10^{14} \mathrm{n} / \mathrm{cm}^{2}$

- online Radiation dose monitoring system measures accumulated ionizing dose in $\mathrm{SiO}_{2}$, displacement damage in silicon and fluences of thermal neutrons.

- continuous monitoring of doses necessary to understand performance of the detector

- doses are monitored at 14 locations in the Inner Detector and at 48 locations at larger radii

- sensors are read out every 60 minutes and readings are stored in the database.

$\rightarrow$ results of dose measurements after 2 years of ATLAS data taking are presented

TID measurements with RadFETs

- RadFETs: p-MOS transistor

- holes caused by radiation get trapped In the gate oxide:

$\rightarrow$ increase of thr

sensitivity and dynamic range depend on

oxide thickness:

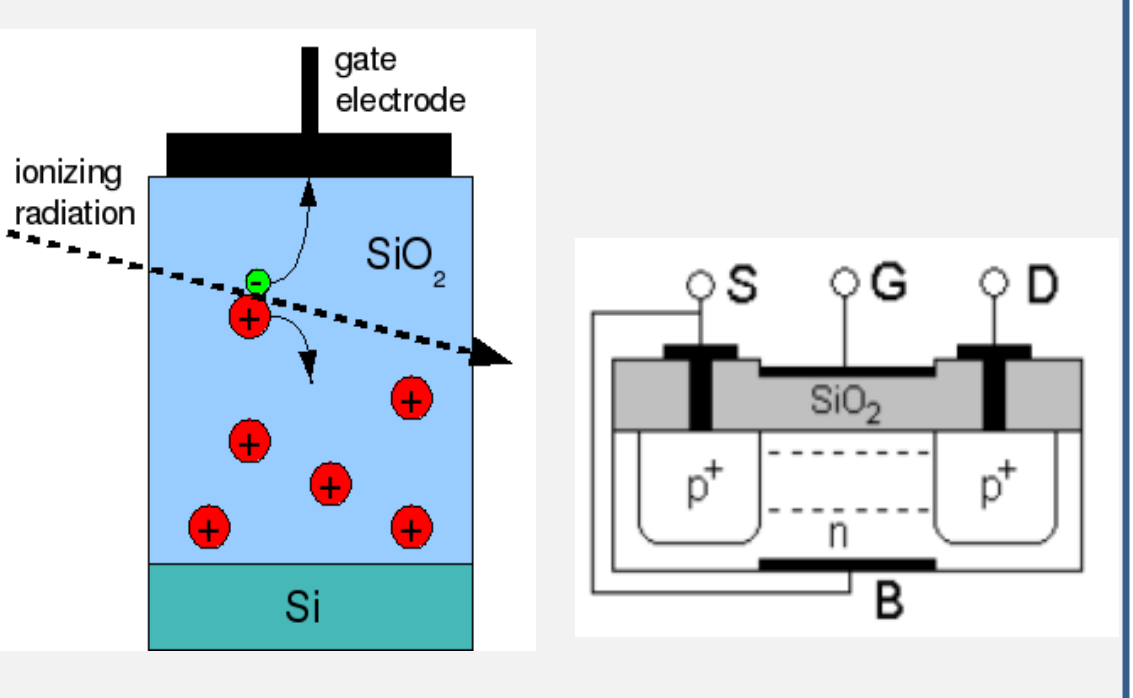

Inner detector:

LAAS $1.6 \mu \mathrm{m} ;$ REM $0.25 \mu \mathrm{m}$

Other locations

$\frac{\text { Other locations }}{\text { - LAAS } 1.6 \mu \mathrm{m}}$

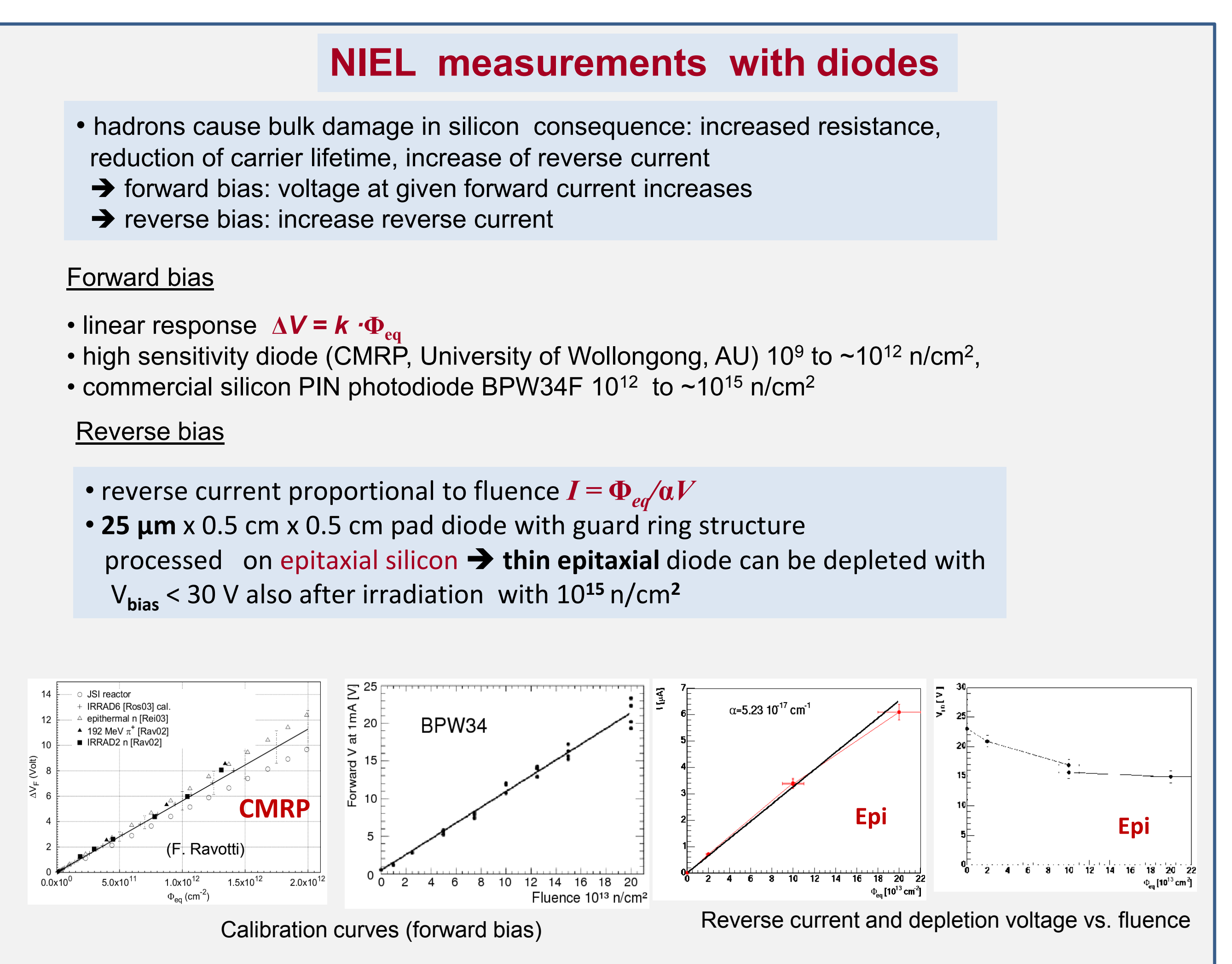

Thermal neutrons

- bipolar transistors (DMILL) used in front end ASICS

measure base current at given collector current

$\rightarrow$ monitor status of front end electronics
$\rightarrow$ sensitive to fast and thermal neutrons

$\Delta \mathrm{I}_{\mathrm{b}} / \mathrm{I}_{\mathrm{c}}=\mathrm{K}_{\mathrm{eq}} \cdot \Phi_{\mathrm{eq}}+\mathrm{K}_{\mathrm{th}} \cdot \Phi_{\mathrm{th}}, \mathrm{K}_{\mathrm{eq}}, \mathrm{K}_{\mathrm{th}}$ and $\Phi_{\mathrm{eq}} \mathrm{known}$
$\rightarrow \Phi_{\mathrm{th}}$ can be determined

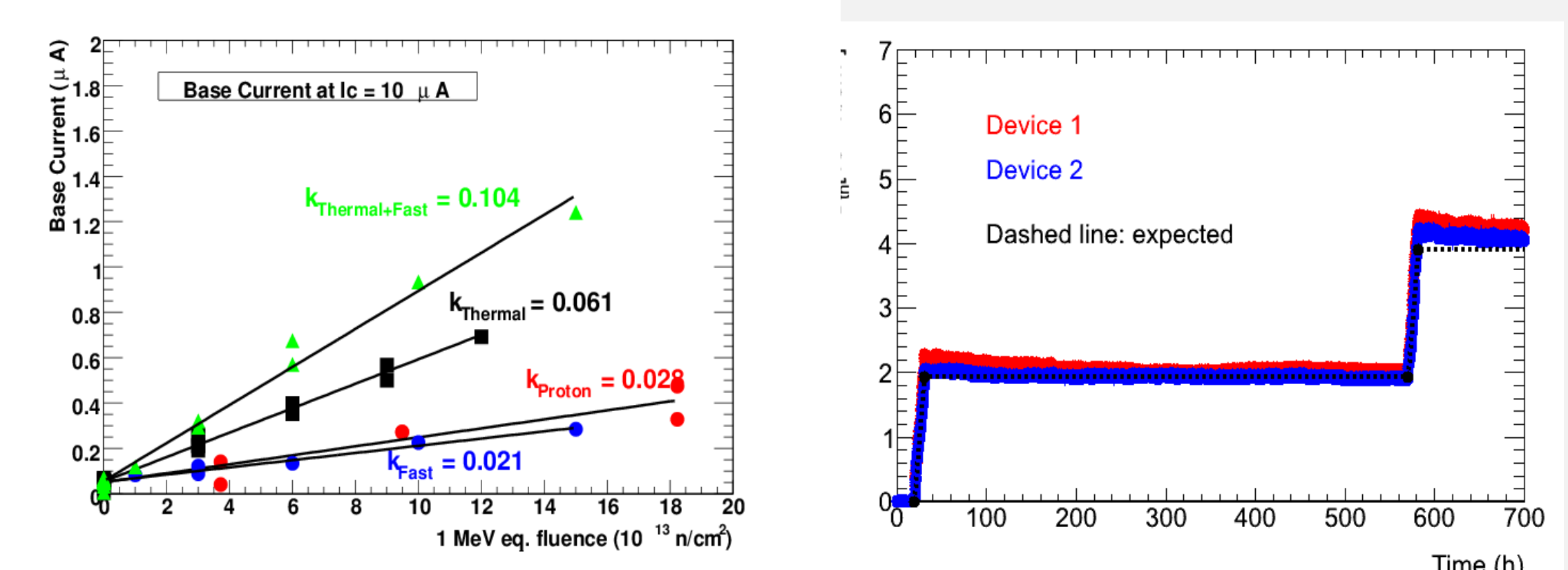
Ast hadrons, thermel neer irradiation with thermal and fast neutrons (reactor)
Inner Detector

arge racess in 10 years

$\rightarrow$ need many sensors
- for dose monitoring in the Inner Detector:

Radiation Monitor Sensor Board (RMSB

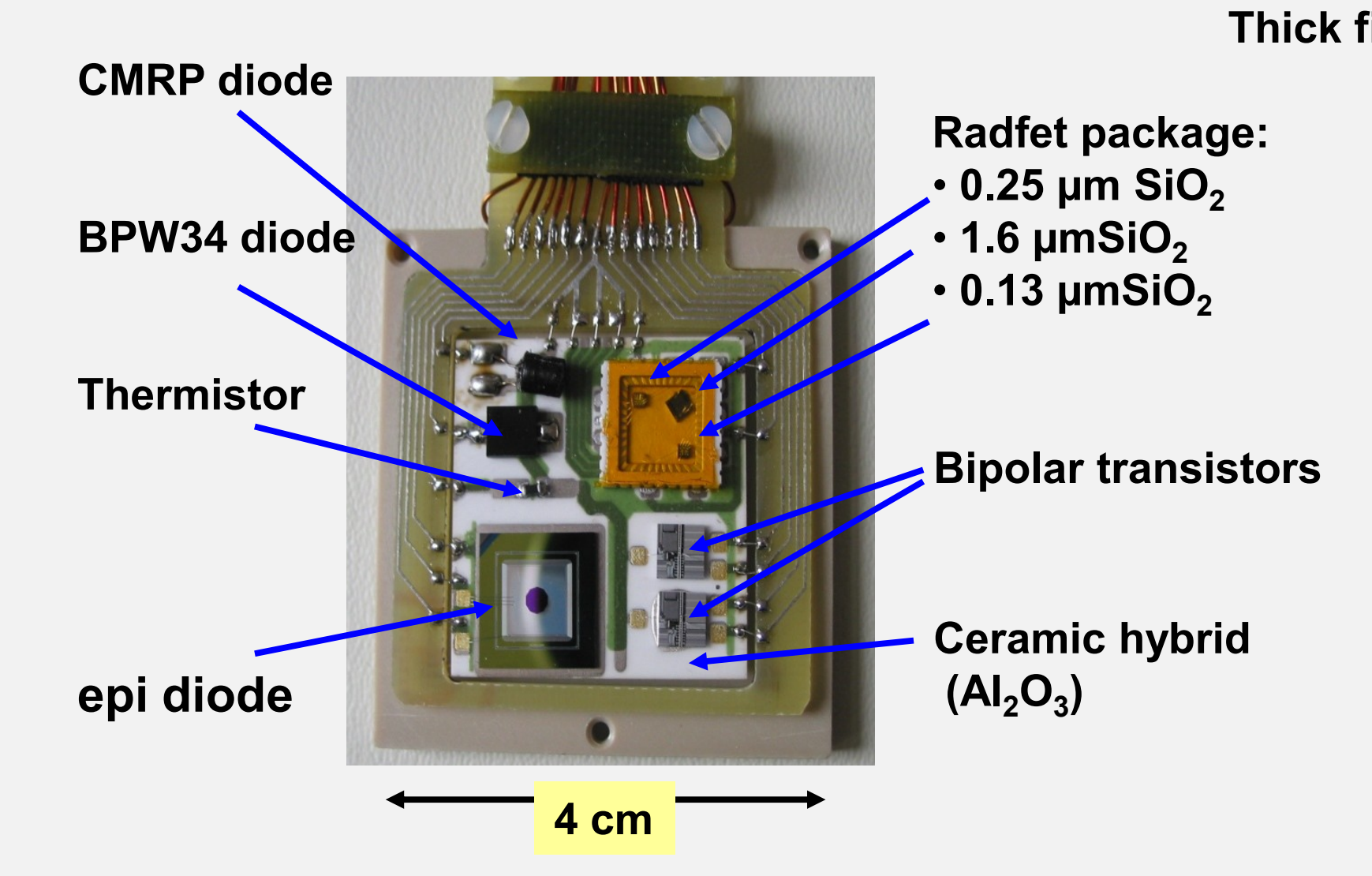

Fluka simulations

Radiation backgrounds have been simulated in the ID using the

- FLUKA particle transport code

- simulations done for $\checkmark s=7 \mathrm{TeV}$ assuming a proton-proton inelastic cross section $77.5 \mathrm{mb}$ as

- ionizing dose in $\mathrm{SiO}_{2}$ and displacement damage in silicon calculated from simulated particle

fluxes

predictions of doses in Gy and fluences of $1 \mathrm{MeV}$ equivalent neutrons normalized to unit of integrated luminosity
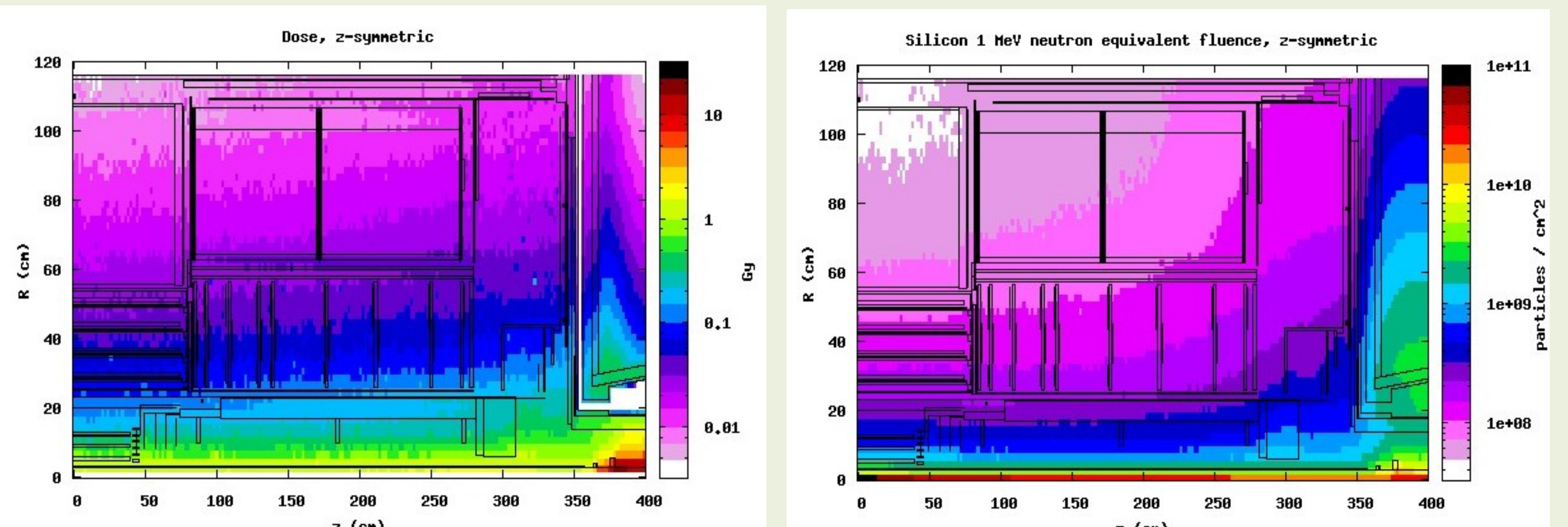

Dose per $\mathrm{pb}^{-1}$ integrated luminosity

$1 \mathrm{MeV}$ equivalent fluence per $\mathrm{pb}-1$

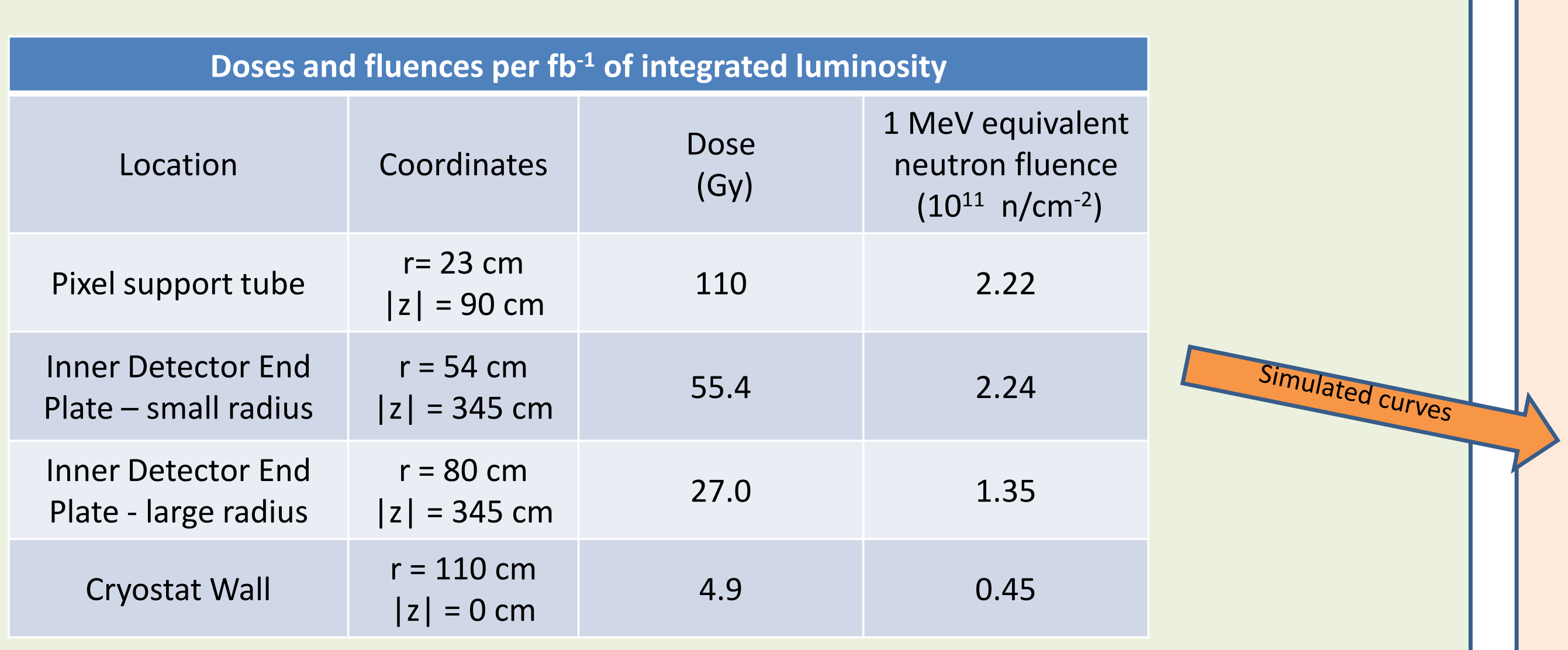

\section{Caloriemeters, Muon detectors}

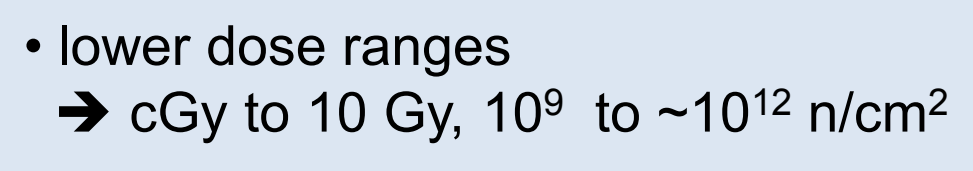

$\rightarrow$ no temperature stabilization
$\rightarrow$ correct read out values with

$\rightarrow$ correct read out values with known
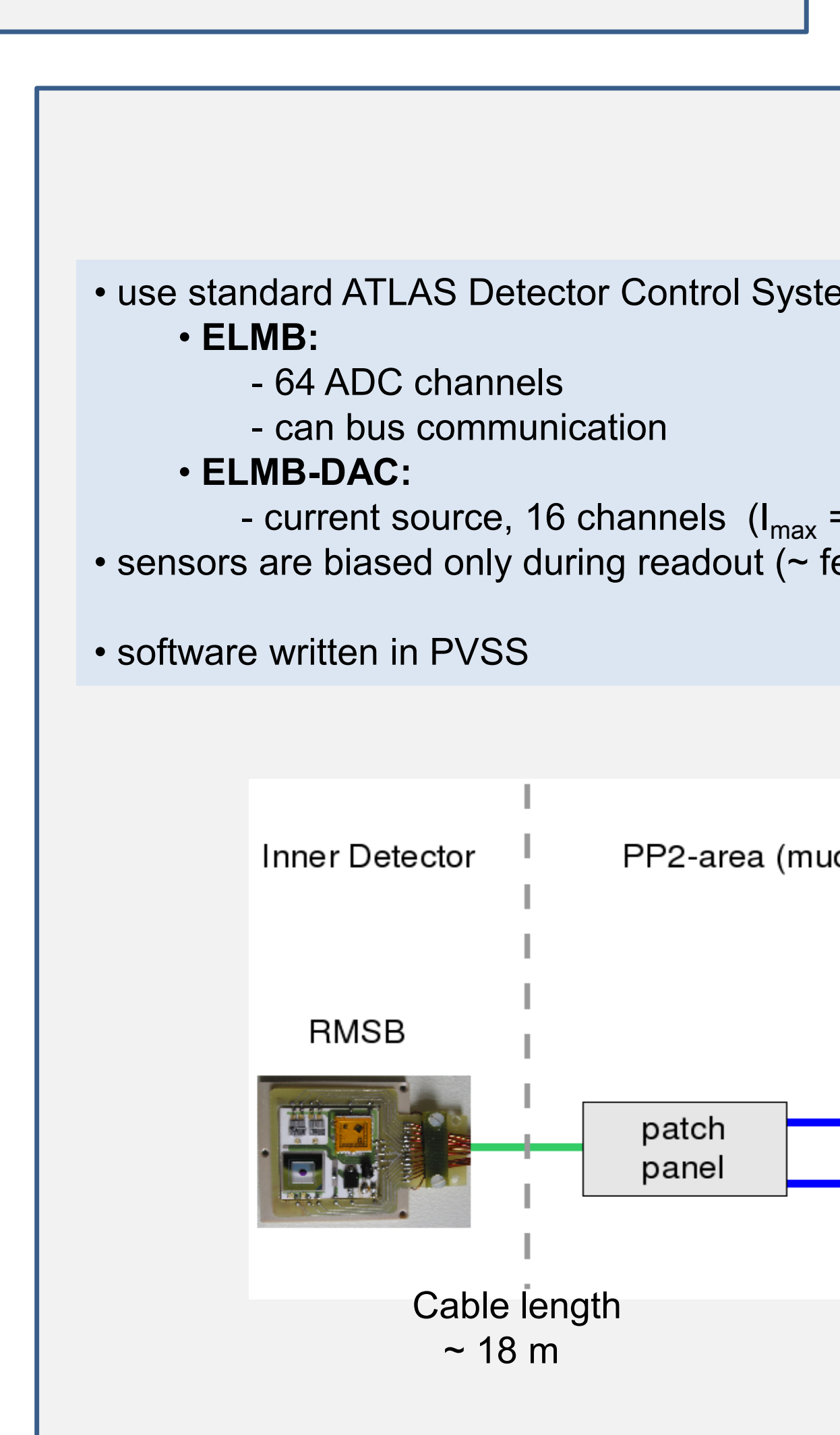

Readout



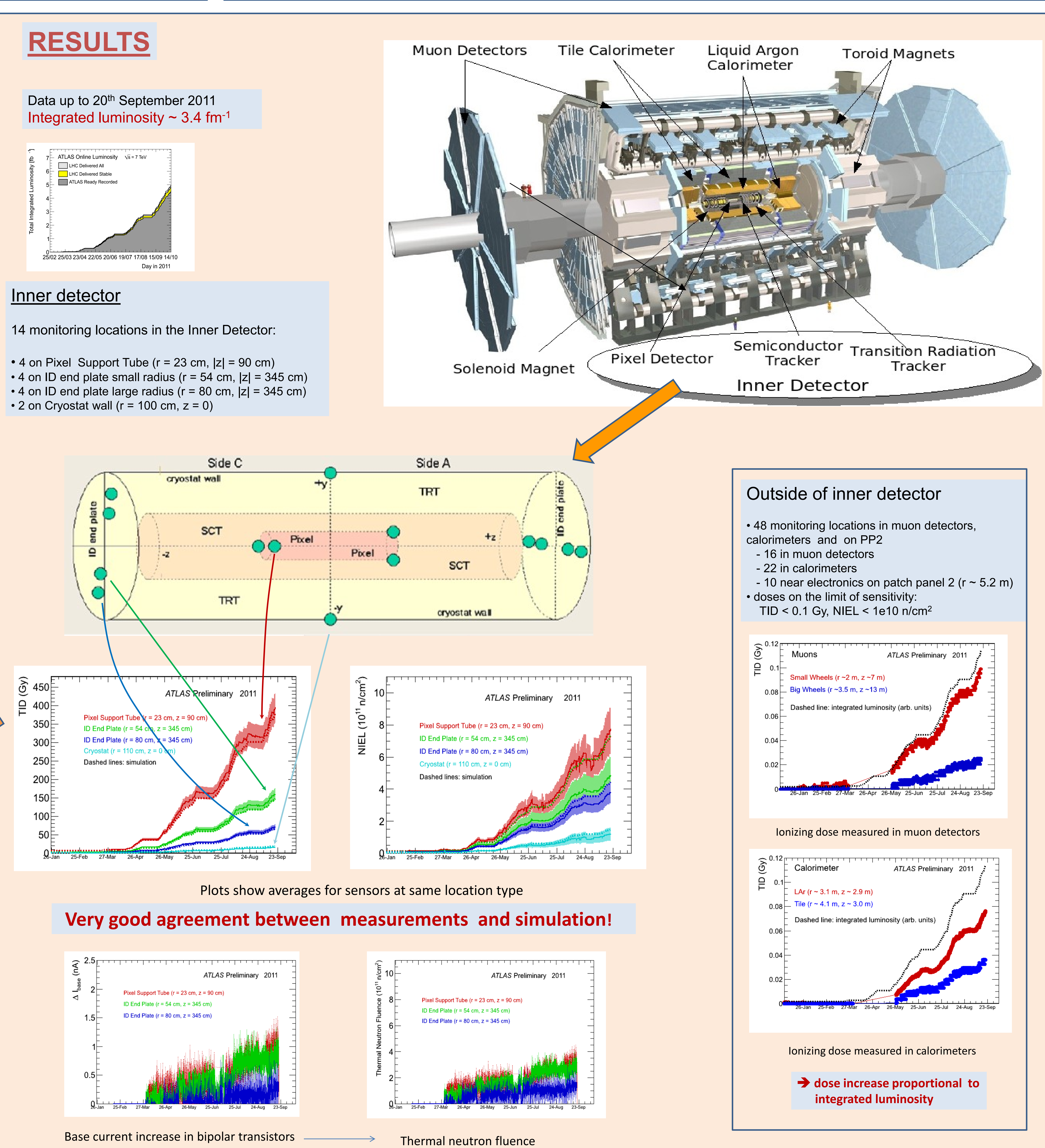

